
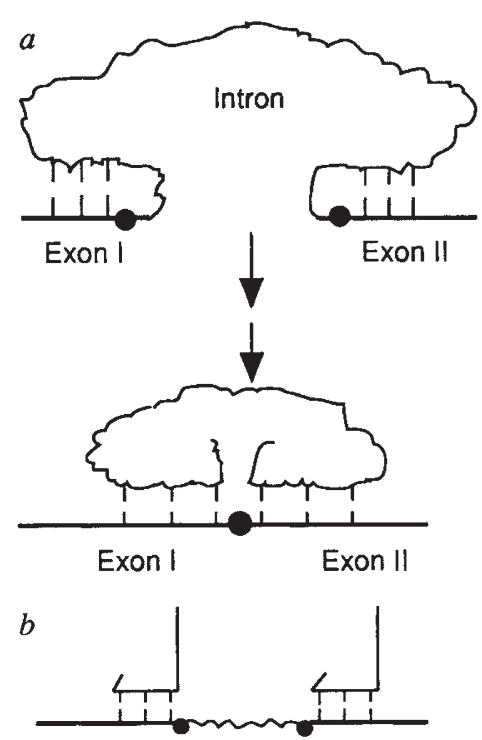

Exon 1 Intron Exon II

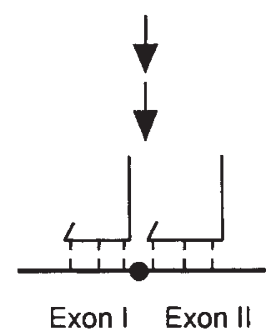

c

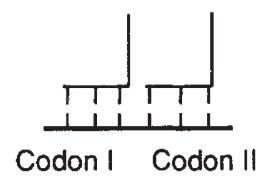

Base-pairing of short sequences flanking splice junctions in putative ancestral RNAs related to $a$, group I introns or $b$, small nuclear RNAs (snRNAs), could have given rise to complexes that are structurally analogous to two tRNAs bound to adjacent codons on a messenger RNA $(c)$. The dots indicate the positions of the splice junctions.

tertiary folding. Davies ${ }^{10}$ has made the provocative suggestion that antibiotics may have co-evolved with RNA to produce the modern ribosome, but the idiosyncratic phylogenetic distribution of antibiotic-producing organisms would support more recent origins. Another possibility that comes to mind is that group I introns might have evolved from ribosomes; the fact that they often interrupt rRNA genes hints at such a possibility.

Most intriguing, however, is the converse idea, that ribosomes evolved, at least in part, from an ancestral RNA related to the group I introns. RNAbased life would almost certainly have made use of genetic recombination, which, in the case of RNA, is commonly known as trans-splicing. Recognition of splice sites (and, in the case of premessenger RNA, branch-point recognition) is usually accomplished by RNA-
RNA base pairing, using short complementary sequences (notable exceptions are pre-tRNA splicing and recognition of the pre-mRNA $3^{\prime}$ splice site, which involve proteins). An interesting consequence is that for a generalized primitive splicing reaction, in which the $5^{\prime}$ and $3^{\prime}$ splice junctions are recognized in this way, the complex at the instant of splicing bears a striking resemblance to two tRNAs bound to mRNA at adjacent codons (see figure). If the transesterification activity of the ribozyme were subverted to catalyse the chemically related acyl-transfer reaction of peptidebond formation, the stage would be set for the evolution of a primitive ribosome. Selective pressure for such a process could have arisen if the efficiency of splicing were improved by attachment of amino acids or peptides to the ribozyme.

Another link between self-splicing and translation comes from the discovery that the group I introns specifically bind arginine $\mathrm{e}^{11}$, which is recognized by the same part of the RNA that binds the guanosine co-factor. Yarus points out that, although the sequence that helps to form the binding site shows considerable phylogenetic variation among the more than one hundred known sequences, it is always centred on a triplet corresponding to one of the arginine codons ${ }^{12}$.

Whether or not these findings turn out to be relics of an ancient connection between RNA splicing and the origins of translation remains to be seen. But even if the binding of antibiotics and amino acids to group I introns turns out to be the result of convergent evolution, the implications are no less significant. The evolution of an RNA with the ability to recognize some of the same small molecules that are also recognized by the translation machinery (and to this list can be added the guanosine co-factor) is, after all, not a rare and improbable event.

Harry F. Noller is at the Sinsheimer Laboratories, University of California, Santa Cruz, California 95064, USA.

1. von Ahsen, U., Davies, J. \& Schroder, R. Nature 353 368-370 (1991)

2. Cundliffe, E. in The Molecular Basis of Antibiotic Action (eds Gale, E. F., Cundliffe, E., Reynolds, P. E., Richmond, M. H. \& Waring, M. H.) 402-547 (Wiley, New York, 1981).

3. Cech, T. R., Zaug, A. J. \& Grabowski, P. J. Cell 27 487-496 (1981)

4. Guerrier-Takada, C., Gardiner, K., Marsh, T., Pace, N. \& Altman, S. Cell 35, 849-857 (1983)

5. Cech. T. R. A. Rev. Biochem. 59, 543-568 (1990)

6. Moazed, D. \& Noller, H. F. Nature 327, 389-394 (1987).

7. Moazed, D. \& Noller, H. F. J. molec. Biol. 211 135-145 (1990)

8. Noller, H. F. A. Rev. Biochem. 60, 191-227 (1991)

9. Davies, J., Gorini, L. \& Davis, B. D. Molec. Pharmac. 1 93-107 (1965)

10. Davies, J. Molec. Microbiol. 4, 1227-1232 (1990)

11. Yarus, M. Science 240, 1751-1758 (1988)

12. Yarus, M. \& Christian, E. L. Nature 342, 349-350 (1990)

\section{Making tracks}

HARD on the heels of their molecular shuttle, P. R. Ashton et al. have produced a molecular train (Angew. Chem. Int. Ed. Engl. 30, 1042-1045; 1991). Both are supramolecular assemblies based on complexes formed between hydroquinones and bipyridinium cations. One of these species will spontaneously thread itself through a cyclic form of the other, and the resulting complex can be readily converted into two linked macrocycles a catenane. Ashton et al. have now threaded a bipyridinium ring (the train) onto a larger hydroquinone ring (the track) with several docking points (the stations). At room temperature the train runs freely around the track, but at about $-60^{\circ} \mathrm{C}$ it sticks at one station. All that remains is to incorporate into the track signalling devices that can direct the traffic.

\section{On labour day}

How does an expectant mother know when to give birth? In the case of sheep at least, the fetus itself signals its readiness for the outside world say $T$. J. McDonald and P.W. Nathanielsz in the American Journal of Obstetrics and Gynecology (165, 764-770; 1991). Normally, just before the onset of labour, characteristic changes occur in the levels of three hormones, adrenocorticotropic hormone and cortisol in the fetus and progesterone in the mother; but these were not seen in cases where a lesion had been made in the paraventricular nucleus of the fetal brain, with the result that pregnancy continued beyond term. How this small region of brain senses and communicates fetal readiness for delivery, and how accurately the sheep model reflects what goes on in humans, are among the remaining questions.

\section{Get knotted}

TAKING the biochemical equivalent of doodling to technical heights, J. E. Mueller, S. M. Du and N. C. Seeman report the synthesis of a trefoil knot using single-stranded DNA (J. Am. chem. Soc. 113, 6306-6308; 1991). The authors allow Watson-Crick hydrogen bonding to do the hard work. The single strand they start with contains two distinct pairs of complementary sequences that naturally twine themselves around one another. Once the molecule is suitably entangled, the authors use T4 DNA ligase to join the $3^{\prime}$ and $5^{\prime}$ ends of the strand to make a single, inextricably knotted loop. They convince themselves that knotted DNA has been made, along with circular and linear molecules, using electrophoresis and sedimentation. As well as producing a three-leafed, singly linked trefoil knot, the authors believe they have made a higher five-leafed, doubly linked form. 